\title{
Physiological Predictors of Reproductive Outcome and Mother-Infant Behaviors in Captive Rhesus Macaque Females (Macaca mulatta)
}

\author{
Allison Cleveland', Gregory C Westergaard*,', Marisa K Trenkle' and J Dee Higley ${ }^{2}$ \\ 'Division of Research and Development, LABS of Virginia, Inc., Yemassee, SC, USA; ${ }^{2}$ Laboratory of Clinical Studies, National Institute on Alcohol \\ Abuse and Alcoholism, USA
}

\begin{abstract}
Previous research has shown that offspring of females with low cerebrospinal fluid (CSF) 5-hydroxyindoleacetic acid (5-HIAA) concentrations are less likely to survive the first year of life than are offspring of females with high CSF 5-HIAA concentrations. In addition, studies of free-ranging rhesus macaque males have suggested that individuals with low CSF 5-HIAA concentrations suffer reduced reproductive success relative to their high serotonin counterparts. We examined CSF concentrations of the monoamine metabolites 5 HIAA and homovanillic acid (HVA), and plasma cortisol concentrations as predictors of first-time adult reproductive potential, maternal behavior, and overall social interactions in two groups of captive female rhesus macaques and their first offspring. Repeated CSF and blood samples were obtained from adult females in two social groups, and focal observations were performed for both new mothers and infants during the first month following parturition. We found that the reproductively aged nulliparous females who failed to give birth to their first offspring showed significantly lower CSF 5-HIAA concentrations than those females who gave birth. Among those females that gave birth to offspring, females with low CSF 5-HIAA concentrations and females with high plasma cortisol concentrations were overly protective and restrictive with their infants. CSF HVA concentration was not associated with reproductive output, social behavior, aggression, or mother-infant interactions in this sample of rhesus macaque females. We conclude that low CNS serotonin activity and high stress, measured by high plasma cortisol, are correlated with reduced reproductive success and patterns of high maternal restrictiveness in young adult female rhesus macaques.
\end{abstract}

Neuropsychopharmacology (2004) 29, 90 I-910, advance online publication, I0 December 2003;. doi: I 0.1038/sj.npp. I 30036 I

Keywords: 5-hydroxyindoleactetic acid; Macaca mulatta; cortisol; stress; maternal behavior; serotonin

\section{INTRODUCTION}

Much attention has been given to the roles that mothers and individual mothering styles play in the social and psychological development of human infants. Research has shown that many of the behavioral and physiological consequences of maternal deprivation and abuse found in humans extend to our closest nonhuman relations (Hinde and Simpson, 1967; Hinde and Spencer-Booth, 1971; Suomi and Ripp, 1983; Bayart et al, 1990; Champoux et al, 1994; Higley et al, 1996b, c; Kraemer and Clarke, 1996; Higley and Linnoila, 1997; Kraemer, 1997; Maestripieri and Carroll, 1998; Higley and Bennett, 1999). This suggests that physiological mechanisms that underlie interindividual variation in

\footnotetext{
*Correspondence: GC Westergaard, Division of Research and Development, LABS of Virginia, Inc., 95 Castle Hall Road, PO Box 557, Yemassee, SC 29945, USA, Tel: + I 843589 5190, ext. 22, Fax: + I 843589 5290, E-mail: GWPRIMATE@netscape.net

Received 16 May 2003; revised I8 August 2003; accepted 0 I October 2003

Published online 10 December 2003 at http://www.acnp.org/citations/ Npp I029030322 I/default.pdf
}

parent-infant interactions among nonhuman primates may be similar to those that underlie interindividual variation in human parental care (Hinde and Simpson, 1967; Hinde and Spencer-Booth, 1971; Bayart et al, 1990; Siegel et al, 1993; Champoux et al, 1994; Boyce et al, 1995; Lubach et al, 1995; Clarke et al, 1996; Kraemer and Clarke, 1996; Kraemer, 1997; Maestripieri and Carroll, 1998; Sanchez et al, 1998).

Rhesus macaque (Macaca mulatta) mothers play an important role in the socialization process and have been especially useful as animal models for the study of maternal behavior and its physiological and environmental correlates (Seay and Harlow, 1965; Suomi, 1973; Meyer et al, 1975; Suomi et al, 1976; Suomi et al, 1978). Mother-infant bonds are an integral component of life even after infants stop nursing, serving as a secure base to reduce fear and providing social support during challenges. Among most Old World primates, in fact, mothers are the primary socializers of their offspring, punishing aberrant behavior and reinforcing positive responses (Maestripieri and Carroll, 1998).

Fairbanks has summarized the literature for nonhuman primates on maternal behavior and has shown that it can be 
categorized into two general characteristics - protectiveness and rejection (Fairbanks and McGuire, 1988; Fairbanks, 1989, 1996). While almost all mothers restrict their infant's movement during the first few days after birth, as the infant matures, mothers vary in how freely they allow them to venture from their immediate presence. By the second and third week of life, most mothers allow their infants significant time in unrestrained exploration and interactions with other group members. A few mothers, however, particularly those whose infants are at risk for injury or harm, remain highly restrictive, maintaining relatively constant physical contact. Other mothers promote independence by rejecting and leaving their infant. Such maternal behavior applied skillfully and at appropriate times is believed to promote independence and selfreliance. On the other hand, overly protective maternal restrictiveness has negative consequences since it limits the infants opportunities to explore and play, principal methods that immature primates use to integrate themselves into the larger group and to acquire the skills to become socially competent (Symons, 1978; Chalmers, 1984). Perhaps the most important consequence is that infants who are restricted from interacting with other conspecifics are limited in their opportunity to form relationships and coalitions (Symons, 1978; Suomi et al, 1981; Smith, 1982; Lee, 1983). Mothers who are overly protective and fail to promote independence by the skillful use of infant rejection produce offspring who are less socially complex, less observant of the immediate environment, and less likely to explore novel stimuli (Fairbanks, 1989).

Maternal protectiveness and rejection both show longterm, trait-like interindividual stability (Fairbanks, 1989; Berman, 1990), and are transmitted across generations. For example, infants who receive high levels of restrictiveness are likely to become adult females who are restrictive of the next generation of offspring (Fairbanks, 1989). Rates of aggression from other group members may also affect mother-infant behavior. For example, Simpson and Howe (1997) found that females that received high levels of aggression were more restrictive of their infants than were mothers who did not receive aggression, and the infants whose mothers received aggression spent less time participating in social interactions with other group members.

There is evidence that biogenic amines play a role in the expression of nonhuman primate mothering behaviors, although their influences on mother-infant interactions and life history parameters are poorly understood. In one group of captive rhesus monkeys (Maestripieri et al, unpublished), cerebrospinal fluid (CSF) concentrations of the serotonin metabolite 5-HIAA and the norepinephrine metabolite MHPG were higher in abusive mothers, who as infants had been abused by their own mothers, when compared to nonabusive mothers. Preliminary data suggest that when compared to nonabused infants these abused infants exhibited low CSF 5-HIAA concentrations. In their study, Maestripieri et al found that abusive mothers exhibited behaviors such as hitting, dragging, dropping, biting, and crushing infants. While we have not observed such extreme abuse in our female population, we have found that females with low CSF 5-HIAA concentrations were more likely to engage in violent aggression, and infants born to these adult rhesus females with low CSF 5-HIAA were more likely to die within a year after birth than were infants of females with high CSF 5-HIAA concentrations (Westergaard et al, 2003a). In addition, Fairbanks et al (1998) found that, among captive vervets, females with low CSF 5-HIAA concentrations were more protective and restrictive of their neonates than were their high CSF 5HIAA counterparts.

In this study, we examined the physiological correlates of mother-infant interactions in captive rhesus macaque females and their first offspring. Specifically, we investigated the relationships among plasma hormones (cortisol and prolactin), monoamine metabolite concentrations (5HIAA and HVA), social behavior, and patterns of motherinfant interactions and proximity in two $M$. mulatta social groups. Given our previous results indicating an increased risk of neonatal mortality for females with low CSF 5-HIAA concentrations, we hypothesized that rhesus mothers with relatively low CSF 5-HIAA concentrations would exhibit less competent maternal social skills as measured by age appropriate maternal restrictiveness and ventral contact (Maestripieri, 1998) relative to high CSF 5-HIAA rhesus mothers. In addition, our previous research has indicated that females with low CSF 5-HIAA concentrations are more likely to be wounded, to engage in dangerous aggression, to be low in social dominance rank (Higley and Suomi, 1996), and less sophisticated socially (Higley et al, 1994; Higley et al, 1996a-c). We therefore hypothesized that females with low CSF 5-HIAA concentrations would be more likely to restrict their infants, eliciting immature clinging and inhibiting social interactions with peers following the first several days after birth. We hypothesized that, if such behaviors are an indication of stress, a similar pattern would occur in females with high plasma cortisol concentrations.

\section{METHODS}

\section{Capture and Housing}

The subjects were 32 female rhesus macaques living under laboratory conditions that approximate the natural environment, born into and transferred from a free-ranging island population of approximately 4500 individuals (Taub and Mehlman, 1989). The animals were approximately 2 years of age at the time of capture. After subjects were removed from the island, the animals were individually quarantined alone in standard stainless steel cages for a period of 12 months while their Herpes-B viral status was assessed, and where they had visual and auditory contact with other subjects. At the end of the quarantine period, these 32 females were found to be B-Virus free and were then placed into small unisexual groups. Following a 1-year period in which animals were housed in unisex social groups, the animals were formed into one of two multimale-multifemale social groups in outdoor field cages that measured $60 \times 60 \mathrm{ft}$. Each field cage contained 16 females and four males. Throughout the investigation, trained personnel performed medical and health observations on a daily basis. 


\section{Subjects}

The subjects were about 5 years of age (mean age $=5.1$ years, range $=4.67-5.17$ years) during the study, housed in their first year of field-cage social group housing. Of the 32 females socially housed, 24 (mean age $=5.1$ years, range $=4.75-5.17$ years) gave birth to offspring during this period. Although the physiological data, collected for all 32 socially housed females, were used to compare physiological variables between those females who gave birth and those who did not, the behavioral data presented here represent $170 \mathrm{~h}$ of continuous focal sampling (mean for each mother $=3.28 \mathrm{~h}$; mean for each infant $=3.26 \mathrm{~h}$ ) for the mothers only. In all, 15 of the infants were female and 10 were male.

\section{Physiological Sampling}

The females were allowed to habituate to their new surroundings and thereafter, we collected five CSF and blood samples at 5-week intervals when the animals were maintained in single cages. Three CSF and two blood samples were obtained during the first 15 months of outdoor field-cage housing, when the animals were between 4.5 and 5.5 years of age. Samples collected during the social group condition include one sample immediately preceding behavioral data collection (November 1995), one sample obtained during the study (April 1996), and one sample following the birth season (November 1996) for CSF sampling. Blood and CSF samples $(3 \mathrm{ml})$ were drawn between 1400 and $1500 \mathrm{~h}$ from the cisterna magna of anesthetized subjects (ketamine hydrochloride, $10 \mathrm{mg} / \mathrm{kg}$, i.m.) using a 22-gauge needle and a $5 \mathrm{~cm}^{3}$ syringe. CSF samples were quick frozen at $-70^{\circ} \mathrm{C}$, and later assayed for 5-HIAA and homovanillic acid (HVA) using high-performance liquid chromatography with electrochemical detection (Scheinin et al, 1983). CSF was removed within $20 \mathrm{~min}$ of ketamine injection. Researchers have found that CSF monoamine concentrations do not increase when the sample is obtained within $30 \mathrm{~min}$ of injection (Bacopoulos et al, 1979; Brammer et al, 1987). We also recorded potential confounds such as animal weight, and time between capture, anesthesia, and CSF sampling. Blood samples were obtained via venipuncture, after which the plasma was frozen and stored at a temperature of $-70^{\circ} \mathrm{C}$. Blood plasma was assayed for cortisol and prolactin by Yerkes Regional Primate Research Center (Atlanta, GA) using radioimmunoassay. Due to technical problems, blood samples were available for only two time points during the field-cage condition. These two samples were obtained during the last two field-cage observations. All inter- and intra-assay coefficients of variation for CSF and hormone assays were $<10 \%$. We recorded animal weights using a commercial scale accurate to $+0.25 \mathrm{~kg}$.

\section{Behavior}

We obtained frequency data using 15-min focal-animal sampling (Altmann, 1974) for both mothers and infants on the 5 th, 10th, 15th, and 20th days following parturition. Subjects were observed for a total of $1 \mathrm{~h}$ each on these days. The general ethogram described grooming and agonistic behaviors, the latter recorded as either low-intensity aggression or high-intensity aggression. Categories for agonism followed those established for rhesus macaques and related species (Altmann, 1962; van Hooff, 1967). The observer noted whether the focal animal was the initiator or recipient of aggression, and these categories were treated separately in our analyses. Displacements, and stationary threats, such as stares, open-mouth threats, head bobs, ground slaps, etc, agonism that was displayed while the subject remained stationary, were scored as low-intensity aggression. Displacements occurred when a subject moved toward another subject and occupied its space while the latter retreated. High-intensity aggression consisted of chases and physical assaults, the former occurring when a subject chased another and it fled (often screaming and exhibiting submission), and the latter occurring for all contact aggression (ie when the subject hit, pushed, slapped, wrestled with, or bit another monkey). Prior to analysis, we converted the absolute frequency of each agonistic an affiliative act into a rate of occurrence for each hour of observation per subject.

In addition to the general ethogram, observers noted the frequencies of the following behaviors during adult female focal observations: mother leaves infant (move to a distance greater than one arm's length, away from subject's own infant), approach infant (move in the direction of the subject's own infant), give groom to the infant (mother moves fingers or lips through the pelage of her infant), restrain infant (physically prohibit infant from moving farther away than one arm's length, usually by holding the infant with one hand), and rebuff infant (mother moves infant away from contact, without physically harming the infant). One arm's length during focal observations was always defined as the mother's arm length. We chose these variables because each indicates some measure of motherinfant proximity and/or affiliation, and have been used to define and describe mothering styles in Old World primates (Fairbanks, 1996). We chose to use a relatively small number of behaviorally and developmentally important behavioral variables, rather than an extensive ethogram. We did this to reduce the chance during data analysis that some comparisons would be significant by chance alone, based on a large number of comparisons.

Durations of clinging and nursing by the infant were recorded during offspring observations. In addition, the frequency of the following behaviors were noted: infant leaves dam (infant ends physical contact with its mother, although it may remain in close proximity), infant approaches its dam (move in a direction toward the mother), infant protest (scored when infant performs 'gecker' vocalization), locomote on mother with no, some, or substantial support (defined as mother providing no hands, one hand, or both hands as support for infant). As with general social behaviors, we converted the absolute frequency of each act into a rate of occurrence for each hour of observation per subject prior to analysis.

\section{Data Analysis}

Absolute concentrations of CSF monoamine metabolites and plasma hormones were converted to standard $z$-scores 
prior to analysis. Pearson's product-moment correlations (Pearson's $r$ ) were used to investigate interindividual stability in monoamine metabolite concentrations across the different housing settings (ie single cage and field cage). In addition, single-cage and field-cage mean $z$-scores were calculated for each CSF variable in each housing condition, providing a mean for both settings for each monoamine metabolite. Unpaired $t$-tests were used to compare interindividual differences in CSF monoamine metabolite and plasma hormone concentrations across housing conditions. Because we were concerned with the immediate effects of monoamine activity on mother-infant behavior, and to maintain consistency in our analyses, we used the fieldcage mean concentration $z$-scores for each of the biochemicals in our statistical analyses. We used Pearson's product-moment correlation tests to examine linear relationships between physiological variables within housing conditions.

Pearson's correlations were used to test for linear relationships between all physiological concentrations and the possible confounding variables that were recorded at sample collection times. Unpaired $t$-tests were used to determine dominance-based differences in physiology and behavioral indices. Dominance rank was coded as either 'high' or 'low', with females in the top half of the hierarchy of each field cage ranking as 'high' and females in the bottom half considered 'low'. Dominance rank was known for 19 animals (10 from one social group and nine from the other). Pearson's correlations were used as initial analyses to test for physiology-based differences in behavior.

Because cortisol was significantly correlated with time to venipuncture and with dominance rank, we used multiple regression analysis to control for these variables when testing the strength of a cortisol-behavior relationship indicated by initial tests. As has been found in most other studies, CSF 5-HIAA and HVA concentrations were highly correlated. Thus multiple regression was used to control for the common correlation between CSF 5-HIAA and HVA in the behavior analyses. Because the gender of the infant has been shown in a number of studies to affect mother-infant interactions, both gender and dominance rank were controlled using multiple regression for all analyses addressing the effects of CSF monoamine metabolite and plasma hormone concentrations on both mothering and infant behavior. Prior to performing analyses, individuals with values more than two standard deviations above or below the mean value for that variable were removed from our analyses.

\section{Observer Reliability}

To establish interobserver reliability, simultaneous observation sessions were conducted periodically throughout the study. During these sessions, two observers concurrently scored the same subject for a 15-min period. The resultant scores were combined in an overall reliability test. The reliability of all behaviors was shown by Cohen's $\kappa$ values to be greater than 0.70 . Observers were blind to all biochemical data and to the specific hypotheses being tested.

\section{RESULTS}

Interindividual Differences in Monoamine Metabolite Concentrations and Plasma Hormones across Time

We first examined relationships between monoamine metabolites and plasma hormone concentrations sampled during single-cage and field-cage housing conditions (field cage only for hormones). Linear analyses indicated that monoamine metabolite concentrations remained stable among individuals within the single-cage housing condition for both CSF 5-HIAA and CSF HVA (average correlations: for CSF 5-HIAA, $r(30)=0.50, p<0.03$; CSF HVA, $r(30)=0.48, p<0.03)$. Within the field-cage housing condition, all physiological variables showed interindividual stability across sampling periods (see Table 1; average correlations: for CSF 5-HIAA, $r(28)=0.44, p<0.05$; CSF HVA, $r(28)=0.41, p=0.05$; for plasma prolactin concentrations, $r(29)=0.37, p<0.05$; for plasma cortisol concentrations, $r(29)=0.52, p<0.01)$.

We formulated both a single-cage and a field-cage mean per individual for each monoamine metabolite variable. Linear analysis indicated that interindividual differences were stable between housing conditions for both of the monoamine metabolite concentrations (for CSF 5-HIAA: $r(30)=0.46, p<0.01$; for CSF HVA: $r(30)=0.67, p<0.001)$.

No significant correlations were found between possible confounding factors and the monoamine metabolites during the single-cage period. During the field-cage sampling period, however, mean cortisol concentrations were significantly negatively correlated with time between capture and venipuncture (see Table 2), and time to sample was thus factored out of all subsequent analyses using

Table I Correlations for Field-Cage Housing Physiological Samples

\begin{tabular}{lcccc}
\hline & Samples & I and 2 & I and 3 & 2 and 3 \\
\hline 5-HIAA & $n=28$ & 0.404 & $0.477 *$ & $0.435 *$ \\
HVA & $n=28$ & 0.353 & $0.394 *$ & $0.476 *$ \\
Cortisol & $n=31$ & $0.524 *$ & NA & NA \\
Prolactin & $n=29$ & $0.373 *$ & NA & NA \\
\hline
\end{tabular}

*Significant at the $95 \%$ confidence level.

$\mathrm{NA}=$ non-applicable.

Table 2 Correlations of Possible Confounding Variables to Physiological Values

\begin{tabular}{lcccc}
\hline Confounding variable & $\begin{array}{l}\text { 5-HIAA } \\
\mathbf{n = 3 1}\end{array}$ & $\begin{array}{c}\text { HVA } \\
\mathbf{n}=\mathbf{3 1}\end{array}$ & $\begin{array}{c}\text { Cortisol } \\
\mathbf{n}=\mathbf{3} \mathbf{1}\end{array}$ & $\begin{array}{r}\text { Prolactin } \\
\mathbf{n}=\mathbf{3 1}\end{array}$ \\
\hline & & & & \\
Day of the week & 0.167 & -0.018 & 0.025 & 0.167 \\
Time to venipuncture & 0.192 & 0.039 & $-0.377 *$ & -0.118 \\
Time to CSF & 0.200 & 0.212 & 0.062 & 0.134 \\
Weight & 0.214 & 0.054 & 0.224 & 0.235 \\
\hline
\end{tabular}

*Significant at the $95 \%$ confidence level. 
cortisol. Additional significant correlations were not observed between physiological measures and our recorded confounding factors.

\section{Relationships among Physiological Variables}

Linear analysis indicated that during the single-cage housing condition, CSF 5-HIAA and HVA concentrations were highly positively correlated $(r(30)=0.680, p<0.0001)$. For field-cage housing, significant correlations were observed only between CSF 5-HIAA and HVA concentrations $(r(30)=0.505, p<0.003)$ and between the plasma prolactin and cortisol concentrations $(r(30)=0.361, p<0.05)$.

\section{CSF 5-HIAA Concentrations and Reproduction}

Our results indicated that females who had offspring during the first year of potential fecundity, in 1996 had higher fieldcage serotonin metabolite concentrations than did females who did not produce offspring in the same year (see Figure 1; mean 5-HIAA concentration per mother $=239.5 \mathrm{vs}$ $215.5 \mathrm{pmol} / \mathrm{ml}$ for nonmothers, $t(29)=2.13, p<0.05)$. CSF HVA, plasma cortisol, and plasma prolactin concentrations did not differ between those females who reproduced in 1996 and those who did not. Hereafter in this section, we include only 1996 mothers in our analyses, as our behavioral data collection protocol was specific to mother-infant interactions.

\section{Monoamine Metabolite Concentrations, Dominance, Aggression, and Social Behavior}

We investigated the relationship between CSF monoamine concentrations and the behaviors of the adult females such as dominance rank, social affiliation, and aggression. No relationship was found with respect to CSF 5-HIAA and social dominance rank (mean for high-ranking females $=232.2$ vs $247.2 \mathrm{pmol} / \mathrm{ml}$ for low-ranking females; $t(17)=-0.80, p=0.43)$, nor with time spent in close proximity to, grooming or receiving groom from other animals. Linear analysis indicated that CSF HVA was also not significantly correlated with these adult female variables. CSF concentrations of 5-HIAA and HVA were not

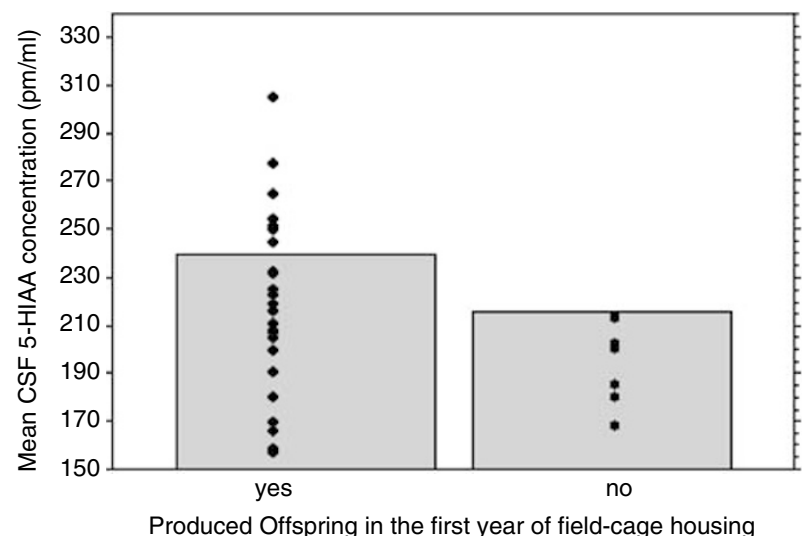

Figure I Bar graph showing differences in mean CSF 5-HIAA concentration $(\mathrm{pm} / \mathrm{ml})$ between those females who produced offspring during the first year of field-cage social group housing $(n=24)$ and those females that did not reproduce $(n=7)$. significantly correlated with the frequency of high-intensity, low-intensity, or escalated aggressive acts, either displayed or received.

\section{Plasma Hormones, Dominance, Aggression, and Social Behavior}

In this group of females, there was a significant positive relationship observed between plasma cortisol levels and dominance ranking. Females of high dominance rank had relatively high plasma cortisol concentrations (mean for high-ranking females $=31.9 v s 26.6 \mu \mathrm{g} / \mathrm{dl}$ for low-ranking females; $t(17)=3.02, p<0.008)$. Rank-related differences were not found with respect to plasma prolactin concentrations. Among these subjects, multiple regression analysis controlling for dominance rank revealed a significant positive correlation between plasma cortisol concentrations and the frequency of low-intensity aggressive acts received $(R(2 / 12)=0.68, p<0.03$; for cortisol: $r=0.65, p<0.02$; for dominance: $r=0.13, p>0.50)$. None of the other behavioral variables were correlated with cortisol or prolactin.

\section{Dominance Rank and Mother-Infant Behavior}

We examined the extent to which dominance ranking was correlated with mother-infant proximity and social interactions. The average amount of time the infants spent clinging to their mothers was positively correlated with mother's dominance rank (mean for infants of high-ranking females $=3015.8$ vs $2752.5 \mathrm{~s} / \mathrm{h}$ for infants of low-ranking females, $t(17)=1.90, p<0.05)$; additionally, the average amount of time infants spent away from their mothers, that is, not clinging, was negatively correlated with rank, with infants of high-ranking mothers spending less time away from their mothers than infants of low-ranking mothers (mean for infants of high-ranking females $=117.6 v s 306.1 \mathrm{~s} / \mathrm{h}$ for infants of low-ranking females, $t(17)=-2.39, p<0.03)$. There was a trend for low-ranking mothers to leave their infants more often than did high-ranking females, but this difference was not significant (mean for high-ranking females $=0.66$ vs $1.55 \mathrm{acts} / \mathrm{h}$ for low-ranking females, $t(17)=-1.37, p=0.18)$. Infants of high-ranking mothers spent significantly more time receiving grooming from other group members than did those of low-ranking mothers (mean for infants of high-ranking females $=43.0$ $v s 8.7 \mathrm{~s} / \mathrm{h}$ for infants of low-ranking females, $t(17)=2.92$, $p<0.01)$. On the other hand, there was a trend for infants of low-ranking females to receive grooming by their own mothers (mean for infants of high-ranking females $=108.8$ vs $192.2 \mathrm{~s} / \mathrm{h}$ for infants of low-ranking females, $t(17)=-1.37, p<0.18)$. Overall, however, the average amount of time infants spent being groomed did not differ between offspring of low- and high-ranking females.

\section{Monoamine Metabolite Concentrations and Mother-Infant Behavior}

During the first 2 weeks, infants spent, on average, more than $84 \%$ of their time on their mothers' ventrum. Analyses for the initial neonatal period, days 5 and 10, indicated no serotonin-based differences in mother-infant proximity patterns, duration of infant clinging, or the amount of time 


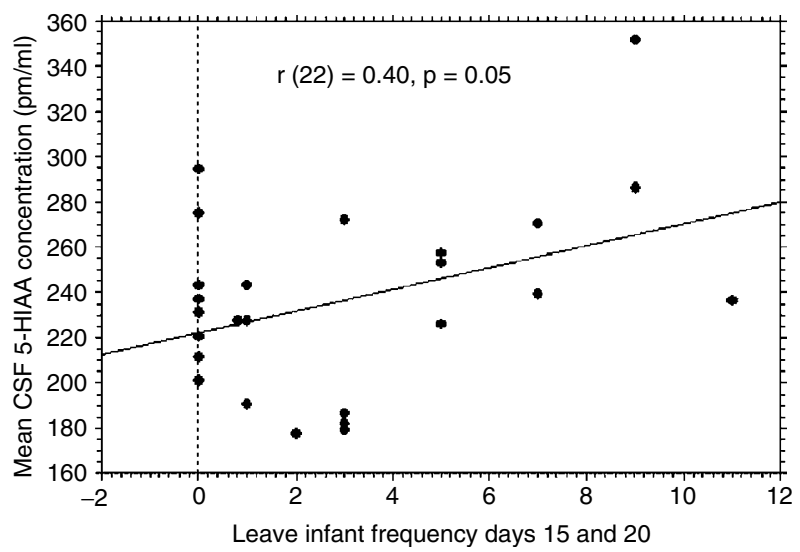

Figure 2 Scatterplot showing the positive correlation between mothers' mean CSF 5-HIAA concentrations $(\mathrm{pm} / \mathrm{ml})$ and the frequency at which mothers were observed leaving their infants (ie, moving out of proximity).

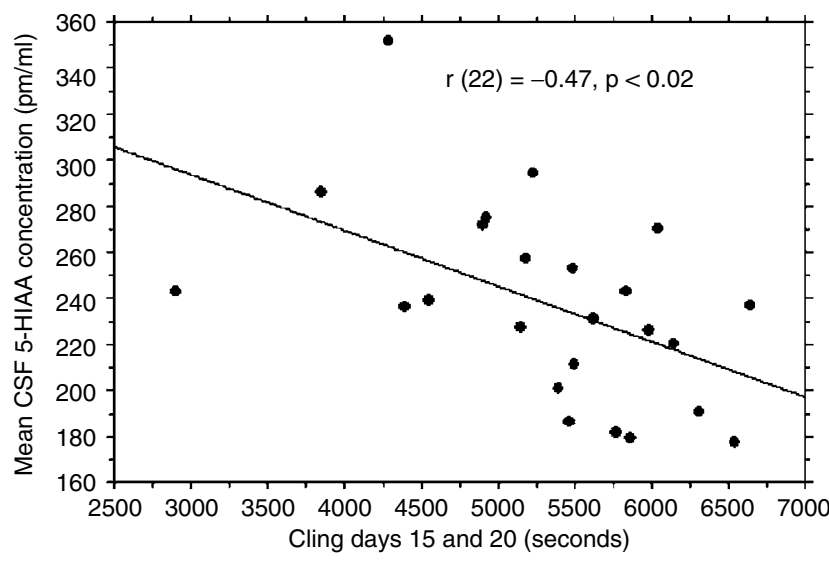

Figure 3 Scatterplot showing the negative correlation between mothers' mean CSF 5-HIAA concentrations $(\mathrm{pm} / \mathrm{ml})$ and the amount of time infants spent clinging to their mothers $(\mathrm{s} / \mathrm{h})$ during days 15 and 20.

infants spent nursing during this initial period. However, as the infants began to explore and gain independence, days 15 and 20 , linear analyses revealed that females with relatively high CSF 5-HIAA left their infants more frequently than did their low serotonin counterparts $(r(22)=0.40, p=0.05$; see Figure 2), although it should be noted that the shared variation for this analysis was low. Perhaps as a reflection of the mothers with high CSF 5-HIAA concentrations showing more rejecting behavior, during the same observation period, infants of females with relatively low CSF 5-HIAA concentrations spent more time clinging than did infants of females with higher CSF 5-HIAA concentrations ( $r$ $(22)=-0.47, p<0.02$; see Figure 3 ). The effect of CSF 5 HIAA concentration on infant clinging during days 15 and 20 remained significant when infant sex and mother's dominance rank were controlled using multiple regression analysis $(R \quad(3 / 14)=0.64, \quad p=0.05$; for CSF 5-HIAA: $r=-0.57, p<0.03$; for dominance: $r=0.30, p=0.20$; for sex: $r=0.26, p>0.20)$. Linear analysis indicated that CSF HVA concentrations were significantly positively correlated with the duration of infant clinging for days 5 and 10, but this correlation was no longer significant when the common correlation between CSF HVA and 5-HIAA concentrations

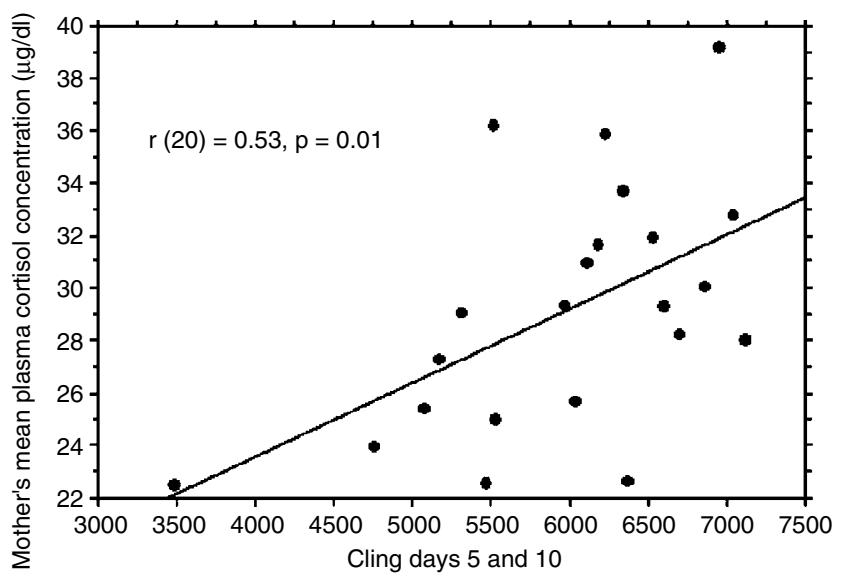

Figure 4 Scatterplot showing the positive correlation between mothers' mean plasma cortisol concentrations $(\mu \mathrm{g} / \mathrm{dl})$ and the amount of time infants spent clinging to their mothers $(\mathrm{s} / \mathrm{h})$ during days 5 and 10.

was controlled for using multiple regression. CSF HVA concentrations were not significantly correlated with other measures of mother-infant proximity, duration of infant clinging, or duration of infant nursing for either period of the study.

\section{Plasma Hormones and Mother-Infant Behavior}

In a multiple regression analysis controlling for mother's dominance rank and infant sex, plasma cortisol levels were positively correlated with the average amount of time infants spent clinging to their mothers during the early neonatal period, days 5 and $10(R(3 / 13)=0.72, p=0.02$; for cortisol, $r=0.89, p<0.01$; for dominance rank, $r=0.23$, $p>0.30$; for sex, $r=0.19, p>0.40$; see Figure 4 ), although it should be noted that the shared variation for this analysis was low. One female with low cortisol and an infant that clung less than most other infants was observed as an outlier. When we performed the same multiple regression analysis with this female removed, our results remained significant $(R(3 / 12)=0.71, p<0.04$; for cortisol, $r=0.82$, $p<0.02$; for dominance rank, $r=0.41, p>0.10$; for sex, $r=-0.09, p>0.70)$. No additional cortisol- or prolactinbased differences in mother-infant proximity patterns, or duration of infant clinging or nursing were observed.

\section{DISCUSSION}

Our results indicate that interindividual differences in CSF 5-HIAA and HVA concentrations were consistent across time and two differing housing conditions in this group of M. mulatta females. This finding replicates and extends our previous work for both male and female macaques (Higley et al, 1996a; Higley and Linnoila, 1997; Lilly et al, 1999; Westergaard et al, 1999), suggesting that for females as well as males, interindividual differences in CSF 5-HIAA concentrations are trait-like, showing consistency across both time and setting. Higley et al (1996a) found that from the single cage to the group setting, interindividual differences are stable and remain stable across settings and over a 2-3 years period. Moreover, in this study, not 
only were females with low CSF 5-HIAA concentrations likely to maintain low CSF 5-HIAA concentrations across time and setting, but low levels in the single cage were predictive of low social dominance rank in the home cage a year later. Over the next 3 years, they were also predictive of excessive violence, as measured by removal for wound treatment or for acting repeatedly as a bully toward the other females in the group (Higley et al, 1996a). HVA was also stable over time and settings. This suggests that monoamine metabolite measures during one sampling session are trait-like, predictive across time and settings. This is important because to the extent that personality, a behavioral trait, or psychopathology is under the influence of the serotonin or dopamine system, the neurotransmittermediated inclination to respond and the intensity with which the response would be exhibited are at least in part constitutionally maintained across settings and time as a result of this trait-like neurotransmitter-mediated response.

As hypothesized, and perhaps one of the most important findings of this study, there was a positive correlation between CSF 5-HIAA concentration and reproductive output in the year-long period of this study (ie monkeys with low CSF 5-HIAA concentrations were less likely to give birth in the first year in which they were of reproductive age). In previous studies among free-ranging male rhesus macaques, low CSF 5-HIAA has been associated with infrequent consort relationships, low copulation rates, and infrequent observable sperm plugs (Mehlman et al, 1997). Within our laboratory, males with relatively low CSF 5HIAA concentrations are less like to produce offspring, irrespective of age (Gerald et al, 2002). The relationships between serotonergic turnover, mating success, offspring production, offspring survivorship, and lifetime reproductive success are currently poorly understood. Within the Gerald et al (2002) study, when we assessed the typical male infertility profile, sperm characteristics were unrelated to serotonin functioning. Previous studies have shown no correlation between reproductive hormones and offspring production (Higley et al, 1996a). None of the females with low CSF 5-HIAA concentrations in this study exhibited any evidence of illness that might explain their failure to conceive, nor was their any marked difference in size that would indicate differences in the rates of maturity. Although full fertility tests were not performed on our females, nor have they to our knowledge been performed on other females with low CSF 5-HIAA concentrations, the decreased rate of offspring in this population of females with low CSF 5-HIAA concentrations may be a result of personality differences in females with low CSF 5-HIAA concentrations.

As described above, previous studies of both free-ranging and laboratory macaques with low CSF 5-HIAA concentrations show them to be lower in social dominance rank, less socially oriented, spending more time alone, grooming less, and having fewer conspecifics in close proximity. As a consequence, when examining the males, we postulated that the lower rates of reproductive success are most likely a result of less competent and less frequent social behavior. However, in this study, female social variables were not statistically correlated with CSF 5-HIAA. A study especially designed to comprehensively address the underlying etiology would be necessary to more directly control for sample variables that might involved. A larger sample size would also be useful, because the current sample is underpowered to detect the relationship between some of our traditional measures such as sociality and serotonin. With a larger sample size, we would be better able to address more fully the variables that affect reproduction. Similarly, CSF 5-HIAA levels were not correlated with escalated aggression, as we have found in previous laboratory studies of adolescent and adult females (Higley et al, 1996a; Westergaard et al, 1999). However, this sample represents only those females that reproduced during their first year of social group housing, and this finding may be a product of a truncated population with higher average CSF 5-HIAA concentrations in this subgroup relative to those females who did not give birth to offspring in the same year. Using our previous studies to estimate power and the sample size necessary to detect serotonin-behavior relationships, it is clear that with a sample size of 25 subjects, we are underpowered to detect such relationships. Additionally, we have found among free-ranging rhesus females that individuals with high CSF 5-HIAA receive aggression less frequently than do individuals with low CSF 5-HIAA concentrations (Westergaard et al, 2003b), and a general reduction of aggression toward this group of females relative to those females who did not reproduce, and thus, were not included in the present analyses, may further complicate the relationship between aggression and serotonergic turnover in this group of subjects.

The results of the present study indicate that mothers with low CSF 5-HIAA concentrations were restrictive of their infant's movements and had infants that spent more time clinging tightly to their mothers' ventrum during days 15 and 20, and mothers with high CSF 5-HIAA concentrations moved out of proximity of their infants more often than did females with low CSF 5-HIAA concentrations. With no correlation between rank and CSF 5-HIAA concentrations, this finding appears to be independent of rank. Moreover, it was high-ranking females who were most restrictive of their infants, and all females were able to retrieve their infants with relative ease when they were being handled by other females, suggesting that this was not a situational effect of the females with low CSF 5-HIAA concentrations being in greater danger of losing their infants. This period of life in the mother-infant relationship is very early in development and is believed by attachment theorists to be the time in which the infant forms the basis of long-term mother-infant attachment bonds (Ainsworth et al, 1978; Bowlby, 1982), with the foundation of secure or insecure attachment bonds being organized during this period. These findings, taken together, suggest that independent of social dominance rank, low serotonergic turnover is associated with high levels of maternal protectiveness. This finding is consistent with the findings of other researchers, such as Fairbanks (1996)who also reported low CSF 5-HIAA concentrations in overly protective mothers when studying vervet monkeys Moreover, to the extent that such maternal restrictiveness persists, it may be that future difficulties are present in the mother-infant relationship that affect the infants' developmental outcome.

In a preliminary study, Lindell et al (1997) found that primiparous females with low CSF 5-HIAA concentrations had difficulties with their infants at a later, critical point in 
the mother-infant relationship, during the weaning period 6 months later; both the neonatal period and weaning periods are times of high stress and strain in the infants' and mothers' adjustment. While long-term outcomes cannot be ascertained from this study, it was clear as we observed these infants from mothers with high CSF 5-HIAA concentrations, who were free to leave and return to their mother, that they enjoyed their interactions and relationships were being formed with the other individuals in the group, perhaps affording them a advantage over the infants from mothers with low CSF 5-HIAA concentrations. There is evidence that the infants from low-serotonin mothers may develop deleterious and possible pathological outcomes. Fairbanks, for example, showed that overly protective mothering behavior was associated with particularly cautious and fearful behavioral outcomes in the infants as they develop (Fairbanks and McGuire, 1988). Moreover, mothers who were overly protective of their offspring produced daughters who were similarly disposed to be overprotective to the next generation of offspring (Fairbanks, 1989). While it is tempting to postulate an experiential treatment effect that leads to such outcomes, it is important to note that low CSF 5-HIAA concentrations are heritable (Higley et al, 1993) and in this study geneenvironment effects cannot be disentangled.

While there was not a significant relationship between CSF 5-HIAA and aggression in this population, the basis of the more protective behavior is in part in differences between mothers' overall rates of received aggression. Our findings showed that infants of females with high plasma cortisol levels were observed clinging for longer periods of time than those of females with lower cortisol levels during the earliest and later neonatal period. Given the additional observation that females with high cortisol received lowintensity aggression more frequently, it may be that the females' protective nature may be in part situational, a method to protect their infants from perceived harm. This interpretation is consistent with findings from Simpson and Howe (1997), who found that high rates of received aggression were correlated with the maintenance of close proximity to infants by mothers. The results of the present study indicate a relationship between received aggression, high cortisol levels, and close mother-infant proximity. In this group, low CSF 5-HIAA levels were also associated with high levels of infant clinging. In our past research of female rhesus macaques, although not in this sample, we have found a negative correlation between CSF 5-HIAA concentrations and aggression received (Westergaard et al, 2003a), and among males with low CSF 5-HIAA concentrations those who were wounded most often were more likely to exhibit high cortisol (Higley et al, 1992). This would be consistent with the pattern found in the present study. Nevertheless, the correlation between CSF 5-HIAA concentrations and close proximity, and between cortisol and close proximity appears to be at least in part independent. Multiple regression using both CSF 5-HIAA concentrations and plasma cortisol shows that close proximity remains statistically significant for the first 10 days and for CSF 5HIAA concentrations it remains statistically significant for the period following, and neither biochemical achieved a level of statistical significance in the period that it was not significant in that before.
The results of this study indicate that females with high plasma cortisol, low serotonin metabolite levels, and frequent received aggression show protective mothering styles. Fairbanks' data show that maternal protectiveness is stable both across time with the same infant and with subsequent infants as well. To the extent that individual differences in the biochemicals studied exhibit an underlying etiology, our findings suggest the potential for stable individual differences in the biochemical systems to underlie the stable temperament differences seen in these mothers (Fairbanks, 1996). Nevertheless, as noted earlier, the ultimate psychological consequence of this pattern is yet poorly understood. Some data suggest other pathological outcomes for the infants of females with low CSF 5-HIAA concentrations. In a recent longitudinal study examining the behavioral and life history correlates of serotonergic activity in female $M$. mulatta, our results indicated that infants of females with low serotonin metabolite concentrations were more likely to die within the first year of life than those of females with higher serotonin (Westergaard et al, 2003a). Two major limitations of the current research include the short duration of the study as well as the captive setting. We are currently collecting data on mother-infant interactions for a sample of new mothers and infants in a large free-ranging colony. The current research follows a group of mothers and infants throughout the entire first year following birth. These data will allow us to make inferences regarding the stability of mothering behavior and mothering style during the course of the neonatal period through weaning, as well as the relationships among monoamine metabolite concentrations, plasma hormone concentrations, and mother-infant interactions over a more extended period, in a more naturalistic setting. Future studies should address more fully the life history consequences of mother-infant interaction patterns, and identify more clearly the ways in which the physiological correlates of these patterns influence life history outcomes in nonhuman primates.

\section{ACKNOWLEDGEMENTS}

This research was conducted with support from NIH Grants 5U42RR05083 and R24 RR09983. The authors wish to thank Alecia Lilly, who supervised and assisted in the day-to-day behavior and biochemical data collection under an NIAAA Intramural Fellowship, and Patrick Mehlman who oversaw LABS support during the data collection phase of the project. In addition, our appreciation is expressed to Stephen Lindell, Courtney Shannon, Anne Hurley, Caroline Brewer, Keri Holmes, and to the LABS Animal Care staff who assisted in the biochemical sampling. The LABS of Virginia, Inc. Institutional Animal Care and Use Committee approved a research protocol for this study in accordance with and as required by the Animal Welfare Act.

\section{REFERENCES}

Ainsworth MDS, Blehar MC, Waters E, Wall S (1978). Patterns of Attachment: A Psychological Study of the Strange Situation. Lawrence Erlbaum Associates: Hillsdale, NJ.

Altmann J (1974). Observational study of behavior: sampling methods. Behavior 49: 227-265. 
Altmann SA (1962). A field study of the sociobiology of rhesus monkeys, Macaca mulatta. Ann NY Acad Sci 102: 338-435.

Bacopoulos NG, Redmond DE, Roth RH (1979). Serotonin and dopamine metabolites in brain regions and cerebrospinal fluid of a primate species: effects of ketamine and fluphenazine. $J$ Neurochem 32: 1215-1218.

Bayart F, Hayashi KT, Faull KF, Barchas JD, Levine S (1990). Influence of maternal proximity on behavioral and physiological responses to separation in infant rhesus monkeys (Macaca mulatta). Behav Neurosci 104: 98-107.

Berman C (1990). Consistency in maternal behavior within families of free-ranging rhesus monkeys: an extension of the concept of maternal style. Am J Primatol 22: 159-169.

Bowlby J (1982). Attachment and Loss: Attachment, Vol 1. Basic Books, Inc.: New York.

Boyce W, Champoux M, Suomi S, Gunnar M (1995). Salivary cortisol in nursery-reared rhesus monkeys: reactivity to peer interactions and altered circadian activity. Dev Psychobiol 28: 257-267.

Brammer GL, Raleigh MJ, McGuire MT, Rubinstein EH (1987). Comparison of ketamine, physical restraint, halothane and pentobarbital: lack of influence on serotonergic measures in monkeys and rats. Neuropharmacology 26: 1615-1621.

Chalmers N (1984). Social play in monkeys: theories and data. In: Smith PK (ed). Play in Animals and Humans. Blackwell: Oxford. pp 119-141.

Champoux M, Suomi SJ, Schneider ML (1994). Temperament differences between captive Indian and Chinese-Indian hybrid rhesus macaque neonates. Lab Anim Sci 44: 351-357.

Clarke AS, Hedeker DR, Ebert MH, Schmidt DE, McKinney WT, Kraemer GW (1996). Rearing experience and biogenic amine activity in infant rhesus monkeys. Biol Psychiatry 40: 338-352.

Fairbanks LA (1989). Early experience and cross-generational continuity of mother-infant contact in vervet monkeys. Dev Psychobiol 22: 669-681.

Fairbanks LA (1996). Individual differences in maternal style: causes and consequences for mothers and offspring. Adv Study Behav 23: 579-611.

Fairbanks LA, McGuire MT (1988). Long-term effects of early mothering behavior on responsiveness to the environment in vervet monkeys. Dev Psychobiol 21: 711-724.

Fairbanks LA, Melega WP, McGuire MT (1998). CSF 5-HIAA is associated with individual differences in maternal protectiveness in vevet monkeys. Am J Primatol 45: 179-180.

Gerald M, Higley S, Lussier ID, Westergaard GC, Suomi SJ, Highley JD (2002). Variation in reproductive outcomes for captive male rhesus macaques (Macaca mulatta) differing in CSF 5-hydroxyindoleacetic acid concentrations. Brain Behav Ecol 60: 117-124.

Higley JD, Bennett AJ (1999). Central nervous system serotonin and personality as variables contributing to excessive alcohol consumption in non-human primates. Alcohol Alcoholism 34: 402-418.

Higley JD, King ST, Hasert MF, Champoux M, Suomi SJ, Linnoila M (1996a). Stability of interindividual differences in serotonin function and its relationship to aggressive wounding and competent social behavior in rhesus macaque females. Neuropsychopharmacology 14: 67-76.

Higley JD, Linnoila M (1997). Low central nervous system serotonergic activity is traitlike and correlates with impulsive behavior. A nonhuman primate model investigating genetic and environmental influences on neurotransmission. Ann NY Acad Sci 836: 39-56.

Higley JD, Linnoila M, Suomi SJ (1994). Ethological contributions: experiential and genetic contributions to the expression and inhibition of aggression in primates. In: Hersen M, Ammerman $\mathrm{RT}$, Sisson L (eds). Handbook of Aggressive and Destructive
Behavior in Psychiatric Patients. Plenum Press: New York. pp $17-32$.

Higley JD, Mehlman P, Taub D, Higley SB, Vickers JH, Suomi SJ et al (1992). Cerebrospinal fluid monoamine and adrenal correlates of aggression in free-ranging rhesus monkeys. Arch Gen Psychiatry 49: 436-441.

Higley JD, Suomi SJ (1996). Effect of reactivity and social competence on individual responses to severe stress in children: investigations using nonhuman primates. In: Pfeffer CR (ed). Intense Stress and Mental Disturbance in Children. American Psychiatric Press, Inc.: Washington, DC. pp 1-69.

Higley JD, Suomi SJ, Linnoila M (1996b). A nonhuman primate model of type II alcoholism? Part 2. Diminished social competence and excessive aggression correlates with low cerebrospinal fluid 5-hydroxyindoleacetic acid concentrations. Alcoholism: Clin Exp Res 20: 643-650.

Higley JD, Suomi SJ, Linnoila M (1996c). A nonhuman primate model of type II excessive alcohol consumption? Part 1. Low cerebrospinal fluid 5-hydroxyindoleacetic acid concentrations and diminished social competence correlate with excessive alcohol consumption. Alcoholism: Clin Exp Res 20: 629-642.

Higley JD, Thompson WT, Champoux M, Goldman D, Hasert MF, Kraemer GW et al (1993). Paternal and maternal genetic and environmental contributions to cerebrospinal fluid monoamine metabolites in rhesus monkeys (Macaca mulatta). Arch Gen Psychiatry 50: 615-623.

Hinde RA, Simpson MJA (1967). Qualities of mother-infant relationships in monkeys. CIBA Found Symp 33: 39-68.

Hinde RA, Spencer-Booth Y (1971). Toward understanding individual differences in rhesus mother-infant interaction. Anim Behav 19: 165-173.

Kraemer GW (1997). Psychobiology of early social attachment in rhesus monkeys. Ann NY Acad Sci 807: 401-418.

Kraemer GW, Clarke A (1996). Social attachment, brain function, and aggression. Ann NY Acad Sci 794: 121-135.

Lee PC (1983). Play as a means for developing relationships. In: Hinde RA (ed). Primate Social Relationships: An Integrated Approach, Vol 1. Sinauer Associates Inc.: Sunderland, MA. pp 82-891.

Lilly AA, Mehlman PT, Higley JD (1999). Trait-like immunological and hematological measures in female rhesus across varied environmental conditions. Am J Primatol 48: 197-223.

Lindell SG, Higley J, Shannon C, Linnoila M (1997). Low levels of CSF 5-HIAA in female rhesus macaques predict mother-infant interaction patterns and mother's CSF 5-HIAA correlates with infant's CSF 5-HIAA. Am J Primatol 42: 129.

Lubach G, Coe CL, Ershler W (1995). Effects of early rearing enivorment on immue responses of infant rhesus monkeys. Brain Behav Immun 9: 31-46.

Maestripieri D (1998). Parenting styles of abusive mothers in group-living rhesus macaques. Anim Behav 55: 1-11.

Maestripieri D, Carroll K (1998). Risk factors for infant abuse and neglect in group-living rhesus monkeys. Psychol Sci 9: 143-145.

Maestripieri D, Megna N, Lindell S, Ayala A, Gold P, Higley J Neurobiological correlates of infant abuse in nonhuman primates and their relation to social and maternal behavior. (unpublished).

Mehlman PT, Higley JD, Fernald BJ, Sallee FR, Suomi SJ, Linnoila M (1997). CSF 5-HIAA, testosterone, and sociosexual behaviors in free-ranging male rhesus macaques in the mating season. Psychiatry Res 72: 89-102.

Meyer JS, Novak MA, Bowman RE, Harlow HF (1975). Behavioral and hormonal effects of attachment object separation in surrogate-peer-reared and mother-reared infant rhesus monkeys. Dev Psychobiol 8: 425-435.

Sanchez M, Hearn E, Do D, Rilling J, Herndon J (1998). Differential rearing affects corpus callosum size and cognitive function of rhesus monkeys. Brain Res 812: 38-49. 
Scheinin M, Chang WH, Jimerson DC, Linnoila M (1983). Measurement of 3-methoxy-4-hydroxyphenylglycol in human plasma with high-performance liquid chromatography using electrochemical detection. Anal Biochem 132: 165-170.

Seay B, Harlow HF (1965). Maternal separation in the rhesus monkey. J Nervous Mental Dis 140: 434-444.

Siegel SJ, Ginsberg SD, Hof PR, Foote SL, Young WG, Kraemer GW et al (1993). Effects of social deprivation in prepubescent rhesus monkeys: immunohistochemical analysis of the neurofilament protein triplet in the hippocampal formation. Brain Res 619: 299-305.

Simpson M, Howe S (1997). Group and matriline differences in the behavior of rhesus monkey infants. Anim Behav 34: 444-459.

Smith PK (1982). Does play matter? Functional and evolutionary aspects of animal and human play. Behav Brain Sci 5: 139-184.

Suomi SJ (1973). Surrogate rehabilitation of monkeys reared in total social isolation. Child Psychol Psychiatry 14: 71-77.

Suomi SJ, Collins ML, Harlow HF, Ruppenthal GC (1976). Effects of maternal and peer separations on young monkeys. J Child Psychol Psychiatry 17: 101-112.

Suomi SJ, Kraemer GW, Baysinger CM, DeLizio RD (1981). Inherited and experiential factors associated with individual differences in anxious behavior displayed by rhesus monkeys. In: Klein DF, Rabkin J (eds). Anxiety: New Research and Changing Concepts. Raven Press: New York. pp 179-199.

Suomi SJ, Ripp C (1983). A history of mother-less mother monkey mothering at the University of Wisconsin Primate Laboratory.
In: Reite M, Caine N (eds). Child Abuse: The Nonhuman Primate Data. Alan R Liss: New York. pp 50-78.

Suomi SJ, Seaman SF, Lewis JK, DeLizio RD, McKinney WT (1978). Effects of imipramine treatment of separation-induced social disorders in rhesus monkeys. Arch Gen Psychiatry 35: 321-325.

Symons D (1978). Play and Aggression: A Study of Rhesus Monkeys. Columbia University Press: New York.

Taub DM, Mehlman PT (1989). Development of the Morgan Island Rhesus Monkey colony. Puerto Rico Health Sci J 8: 159-169.

van Hooff JARAM (1967). The facial displays of the catarrhine monkeys and apes. In: Morris D (ed). Primate Ethology. Aldine Publishing Company: Chicago. pp 7-68.

Westergaard GC, Cleveland A, Trenkle MK, Lussier ID, Higley JD (2003a). CSF 5-HIAA concentration as an early screening tool for predicting significant life history outcomes in female specificpathogen-free (SPF) rhesus macaques (Macaca mulatta) maintained in captive breeding groups. J Med Primatol 32: 95-104.

Westergaard GC, Mehlman PT, Shoaf SE, Suomi SJ, Higley JD (1999). CSF 5-HIAA and aggression in female primates: species and interindividual differences. Psychopharmacology 146: $440-446$.

Westergaard GC, Suomi SJ, Chavanne T, Houser L, Hurley A, Cleveland A et al (2003b). Physiological correlates of aggression and impulsivity in free-ranging female primates. Neuropsychopharmacology 28: 1045-1055. 